University of South Carolina

Scholar Commons

$1-21-2009$

\title{
Disentangling Forms of Lorentz Violation With Complementary Clock Comparison Experiments
}

Brett David Altschul

altschul@mailbox.sc.edu

Follow this and additional works at: https://scholarcommons.sc.edu/phys_facpub

Part of the Physics Commons

Publication Info

Published in Physical Review D, Volume 79, Issue 6, 2009.

(C2009 American Physical Society

This Article is brought to you by the Physics and Astronomy, Department of at Scholar Commons. It has been accepted for inclusion in Faculty Publications by an authorized administrator of Scholar Commons. For more information, please contact digres@mailbox.sc.edu. 
arXiv:0901.1870

\title{
Disentangling Forms of Lorentz Violation With Complementary Clock Comparison Experiments
}

\author{
Brett Altschul] \\ Department of Physics and Astronomy \\ University of South Carolina \\ Columbia, SC 29208
}

\begin{abstract}
Atomic clock comparisons provide some of the most precise tests of Lorentz and CPT symmetries in the laboratory. With data from multiple such experiments using different nuclei, it is possible to constrain new regions of the parameter space for Lorentz violation. Relativistic effects in the nuclei allow us to disentangle forms of Lorentz violation which could not be separately measured in purely nonrelativistic experiments. The disentangled bounds in the neutron sectors are at the $10^{-28} \mathrm{GeV}$ level, far better than could be obtained with any other current technique.
\end{abstract}

\footnotetext{
${ }^{1}$ baltschu@physics.sc.edu
} 
There is a great deal of current interest in the possibility that Lorentz and CPT invariances may not be exact in nature. Violations of these symmetries could be tied to quantum gravity, and if any such violation were observed experimentally, it would be a discovery of profound importance. Modern tests of Lorentz and CPT symmetry have included studies of matter-antimatter asymmetries for trapped charged particles [1, 2, 3] and bound state systems [4, 5], determinations of muon properties [6, 7, 8], analyses of the behavior of spin-polarized matter [9, 10], Michelson-Morley experiments [11, 12, 13], measurements of neutral meson oscillations [14, 15, 16, 17, 18, 19], polarization measurements on the light from distant galaxies [20, 21, 22, 23], high-energy astrophysical tests [24, 25, 26, 27], and others. So far, no significant evidence of Lorentz violation has been found.

There is a parameterization of Lorentz and CPT violations in low-energy effective field theory, known as the standard model extension (SME). The SME contains possible Lorentz- and CPT-violating corrections to the standard model [28, 29] and general relativity [30]. The SME provides a useful framework for interpreting experimental tests of these symmetries. Many of the coefficients that characterized the SME have been constrained very tightly. However, many others have not. (Up-to-date information about bounds on SME coefficients may be found in [31.) Moreover, in many cases, only specific combinations of coefficients can be bounded, rather than the individual coefficients themselves.

Some of the most precise laboratory tests of Lorentz symmetry are clock comparison experiments [32, 33, 34, 35, 36, 37]. The results of these experiments are usually interpreted in the context of the Schmidt model [38], which assigns all of a nucleus's angular momentum to a single unpaired nucleon. Since these atomic clock experiments are so powerful, there has been a great deal of interest in devising methods to expand the scope of the bounds they provide. For example, the suggestion that atomic clock experiments on orbiting satellites could add sensitivity to previously unconstrained SME parameters [39] attracted a great deal of interest. In this paper, we shall show that with multiple complementary clock comparison experiments using different isotopes, there is unexpected sensitivity to new areas of the SME parameter space. This fact provides a new motivation for experimenters to improve the atomic clock constraints on many SME coefficients, even those for which there are already strong bounds. (A somewhat similar technique, using complementary experiments with different materials, was used in [40] to place improved bounds on an entirely different collection of SME coefficients.)

The minimal SME Lagrange density for a single species of fermion is

$$
\mathcal{L}_{f}=\bar{\psi}\left(i \Gamma^{\mu} \partial_{\mu}-M\right) \psi,
$$

where

$$
\begin{aligned}
M & =m+\not d-\not \gamma \gamma_{5}+\frac{1}{2} H^{\mu \nu} \sigma_{\mu \nu} \\
\Gamma^{\mu} & =\gamma^{\mu}+c^{\nu \mu} \gamma_{\nu}-d^{\nu \mu} \gamma_{\nu} \gamma_{5}+e^{\mu}+i f^{\mu} \gamma_{5}+\frac{1}{2} g^{\lambda \nu \mu} \sigma_{\lambda \nu} .
\end{aligned}
$$


Bounds based on atomic clock and other nonrelativistic experiments are conventionally quoted in terms of specific combinations of SME coefficients, such as $\tilde{b}_{J}=b_{J}-\frac{1}{2} \epsilon_{J K L} H_{K L}-$ $m\left(d_{J T}-\frac{1}{2} \epsilon_{J K L} g_{K L T}\right)$. (The full set of combinations, which are expressed in sun-centered coordinates, is given in [41, 31]. We shall concentrate on the $\tilde{b}$ combination, because it is the best measured in most sectors.) The combinations mix coefficients from $M$ with mass dimension 1 with coefficients from $\Gamma$, which are dimensionless and so must be multiplied by $m$ to have the dimensions match.

In strictly nonrelativistic experiments, the separate coefficients cannot be further disentangled. Despite this, a number of coefficients can in fact be separated, using only existing data from laboratory experiments. (The newly bounded coefficients are not the same as the $\tilde{b}_{J}^{*}$ coefficients, which were introduced in [31] and are the analogues of the $\tilde{b}_{J}$ coefficients for experiments performed with anti-fermions.) What makes the new bounds possible is a small relativistic effect. Because the effect is weak, there is a loss of precision in the resulting bounds, compared with the bounds on the conventional combinations. Nevertheless, these bounds are important, because they represent separate constraints on groups of coefficients that it was not previously believed possible to separate.

Nonrelativistically, the effects of, say, $b_{j}$ and $d_{j 0}$ are equivalent, because $d_{j 0}$ enters the Lagrange density in the form $-\bar{\psi}\left(E d_{j 0}\right) \gamma^{j} \gamma_{5} \psi=-\bar{\psi}\left(m d_{j 0}\right) \gamma^{j} \gamma_{5} \psi$, which has the same structure as the $b$ term in $\mathcal{L}$. However, the nonrelativistic approximation $E=m$ never holds exactly. In particular, a proton or neutron inside a nucleus has an energy smaller than its mass, because of nuclear binding. If the dominant contribution to the measured Lorentz-violating effects in a atomic species comes from a single unpaired nucleon moving in the scalar potential of the nucleus, and the binding energy associated with this nucleon is $e$, what will be bounded is not $\tilde{b}_{j}$, but rather

$$
\stackrel{\circ}{b}_{J}(e)=b_{J}-\frac{1}{2}\left(1+e_{K} / 3 m\right) \epsilon_{J K L} H_{K L}-\left(m-e-2 e_{K} / 3\right) d_{J T}+\frac{1}{2}\left(m-e-e_{K}\right) \epsilon_{J K L} g_{K L T} .
$$

$e_{K}$ is the nucleon kinetic energy, and both $e$ and $e_{K}$ are assumed to be small compared with $m$. We shall neglect $e_{K}$, although its effects are not that strongly suppressed compared with those of $e$. With more data, $e_{K}$ could be used to further disentangle $H$ and $g$.

The $\tilde{b}_{J}$ coefficients are the combinations of SME parameters that appear in the nonrelativistic Hamiltonian for the fermions [42], which can be derived from the relativistic theory by a Foldy-Wouthuysen transformation [43]. However, this transformation process changes in the presence of interactions. If one instead considers fermions moving in a potential well, different effective coefficients such as $\grave{b}_{J}$ will appear.

This makes it possible to distinguish contributions from $M$ terms from those of $\Gamma$ terms. If two experiments are done, using nucleons with binding energies $e_{1}$ and $e_{2}$, and they produce identical bounds, conventionally expressed as $\left|\tilde{b}_{J}\right|<A$, this actually implies separate bounds $\left|b_{J}-\frac{1}{2} \epsilon_{J K L} H_{K L}\right|<\frac{2 A m}{\left|e_{1}-e_{2}\right|}$ and $\left|d_{J T}-\frac{1}{2} \epsilon_{J K L} g_{K L T}\right|<\frac{2 A}{\left|e_{1}-e_{2}\right|}$. Because the nuclear binding makes a small contribution to the overall energies of the nucleons involved, the bounds are worse than the conventionally quoted bounds on $\tilde{b}_{J}$ by a factor 
of $\mathcal{O}\left(m /\left|e_{1}-e_{2}\right|\right)$. On the other hand, in experiments involving relativistic motion, in which energies are large compared with the particle mass, the effects of $M$ and $\Gamma$ terms can be disentangled without any loss of precision. For example, the maximum achievable velocity for a particle (which need not be the speed of light when there is Lorentz violation) generally depends on the $\Gamma$ terms but not on $M$. However, truly relativistic experiments have not been nearly as precise as atomic clocks experiments. The most precise relativistic Lorentz test, which disentangled several contributions to the muon $\tilde{b}_{J}^{\mu}$, gave bounds at the $10^{-24} \mathrm{GeV}$ level [8].

As noted in [33, many clock experiments will have sensitivities to additional SME coefficients, beyond those indicated by the Schmidt model. To understand the additional sensitivities, an improved understanding of nuclear structures would be required. However, this is a complementary effect to the one considered here. Any further sensitivities of the type discussed in [33] would still be sensitivities to the conventional combinations of coefficients and would not allow the disentanglement of $M$ and $\Gamma$ terms.

The two best atomic clock experiments which measured neutron Lorentz violations involved ${ }^{3} \mathrm{He}$ co-located with heavier species. The heavy species were used as co-magnetometers, while the ${ }^{3} \mathrm{He}$ Zeeman frequencies were read out. The experiments were ultimately sensitive to the combinations $\delta \nu_{\mathrm{He}}-\frac{\mu_{\mathrm{He}}}{\mu_{M}} \delta \nu_{M}$, where the $\delta \nu$ and $\mu$ denote frequency shifts and magnetic moments, and the subscript $M$ indicates the co-magnetometer species.

One experiment used a ${ }^{129} \mathrm{Xe} /{ }^{3} \mathrm{He}$ maser. Taking into account the distinct binding energies $e_{\mathrm{He}}$ and $e_{\mathrm{Xe}}$ of the neutrons in the isotopes involved, the measurements reported in 35. become

$$
\begin{aligned}
\left|\frac{\mu_{\mathrm{He}}}{\mu_{\mathrm{He}}-\mu_{\mathrm{Xe}}} \stackrel{\circ}{x}_{X}^{n}\left(e_{\mathrm{Xe}}\right)-\frac{\mu_{\mathrm{Xe}}}{\mu_{\mathrm{He}}-\mu_{\mathrm{Xe}}} \stackrel{\circ}{x}_{X}^{n}\left(e_{\mathrm{He}}\right)\right|=(-2.2 \pm 7.9) \times 10^{-32} \mathrm{GeV} \\
\left|\frac{\mu_{\mathrm{He}}}{\mu_{\mathrm{He}}-\mu_{\mathrm{Xe}}} \stackrel{\circ}{Y}_{Y}^{n}\left(e_{\mathrm{Xe}}\right)-\frac{\mu_{\mathrm{Xe}}}{\mu_{\mathrm{He}}-\mu_{\mathrm{Xe}}} \stackrel{\circ}{Y}_{Y}^{n}\left(e_{\mathrm{He}}\right)\right|=(8.0 \pm 9.5) \times 10^{-32} \mathrm{GeV}
\end{aligned}
$$

neglecting small contributions from other coefficients.

The other experiment used $\mathrm{K}$ for the co-magnetometer. The magnetic moment of $\mathrm{K}$ is $\mu_{\mathrm{K}} \approx \mu_{B}$, three orders of magnitude greater than $\mu_{\mathrm{He}}$, so the sensitivity to Lorentz violation comes almost entirely from the ${ }^{3} \mathrm{He}$, provided there are no contributions from the $\mathrm{K}$ valance electron. But the relevant type of electron Lorentz violation is ruled out very strongly by torsion pendulum experiments [10]. So the results from this experiment were 37

$$
\begin{aligned}
& \left|\hat{b}_{X}^{n}\left(e_{\mathrm{He}}\right)\right|=(-3.7 \pm 8.1) \times 10^{-32} \mathrm{GeV} \\
& \left|\hat{b}_{Y}^{n}\left(e_{\mathrm{He}}\right)\right|=(-9.0 \pm 7.5) \times 10^{-32} \mathrm{GeV}
\end{aligned}
$$

Each of the four measurements (5-8) implies a $1 \sigma$ bound at the $2 \times 10^{-31} \mathrm{GeV}$ level or better. 
In setting these bounds, numerous approximations have been made, and the bounds cannot be expected to have better than order of magnitude accuracy. The Schmidt model is a significant idealization, and essentially all nuclear interaction effects except the binding energy have been ignored. In reality, the nuclear angular momentum is divided up among protons and neutrons with different binding energies. The correct value of $e$ must be a weighted average of these - and this assumes that it is possible to assign a binding energy to each individual nucleon, which is not strictly the case. The neglect of $e_{K}$ is also a significant approximation.

However, assuming there are no special cancellations between the $\dot{b}_{J}$ coefficients for different species, the $2 \times 10^{-31} \mathrm{GeV}$ bound noted above should be approximately valid. One might be further tempted to conclude that the individual $b, H, d$, and $g$ coefficients that make up $\tilde{b}$ should also be bounded at roughly this level. There is a crucial difference, however, between possible cancellations among the Lorentz violation coefficients for different species and between the various coefficients that make up combinations such as $\tilde{b}_{J}^{n}$. The relative sizes of the contributions to an observable frequency shift made by the SME coefficients for different particles are determined by the complex structures of atoms and nuclei. Even if the various SME coefficients were all comparable in size, it would be remarkable if their contributions cancelled out. On the other hand, the relative sizes of the $M$ and $\Gamma$ coefficients that appear in $\tilde{b}_{J}$ are set by the unknown underlying physics that controls the Lorentz violation, and it would not be unreasonable for a cancellation to exist within $\tilde{b}_{J}$.

For example, consider a model in which a single dimensionless vector field acquires a Lorentz-violating expectation value $u^{\mu}$. (This is known as a "bumblebee" model.) The $b$ and $d$ coefficients for a given species of fermions could then be $b^{\mu}=G m u^{\mu}$ and $d^{\nu \mu}=G u^{\mu} u^{\nu}$, where $G$ is a coupling constant that controls the strength of the interaction between the fermion field and the background. This coupling would generally depend on the species. So there would be a natural relationship between the $b$ vectors for different species - they would all be parallel - but their magnitudes could differ substantially. However, if the expectation value $u^{\mu}$ is a null vector and $u^{\mu}=(1, \hat{u})$ in a frame (such as the rest frame of the cosmic microwave background) that is moving nonrelativistically with respect to the sun-centered frame, $b_{J}-m d_{J T} \approx 0$. This demonstrates that cancellation among the coefficients that make up $\tilde{b}_{J}$ is an entirely natural possibility.

With this in mind, (518) imply

$$
\left|b_{J}^{n}-\frac{1}{2} \epsilon_{J K L} H_{K L}^{n}\right|,\left|m d_{J T}^{n}-\frac{1}{2} \epsilon_{J K L} m g_{K L T}^{n}\right|<\left(\frac{2 m}{e_{\mathrm{Xe}}-e_{\mathrm{He}}}\right)\left(2 \times 10^{-31} \mathrm{GeV}\right)
$$

for $J=X, Y$. With more data, and including $e_{K}$, we could expect to bound $b, H, d$, and $g$ separately at nearly the same level. It remains to determine the difference in the binding energies of the ${ }^{3} \mathrm{He}$ and ${ }^{129} \mathrm{Xe}$ neutrons. ${ }^{3} \mathrm{He}$ is a relatively compact nucleus, with all three nucleons in primarily $1 \mathrm{~S}$ orbital states. The binding energy of $6.69 \mathrm{MeV}$ is nearly evenly distributed among the constituents, giving $e_{\mathrm{He}}=2.23 \mathrm{MeV}$. The case of ${ }^{129} \mathrm{Xe}$ is slightly 
trickier. This isotope has an average binding energy of $8.21 \mathrm{MeV}$ per nucleon. However, the neutron whose spin properties the experiment can observe is a valance neutron and hence less bound than this average would suggest. As a conservative order of magnitude estimate, we shall therefore take $e_{\mathrm{Xe}}-e_{\mathrm{He}} \approx 4 \mathrm{MeV}$. This yields the final bounds

$$
\left|b_{J}^{n}-\frac{1}{2} \epsilon_{J K L} H_{K L}^{n}\right|,\left|m d_{J T}^{n}-\frac{1}{2} \epsilon_{J K L} m g_{K L T}^{n}\right|<10^{-28} \mathrm{GeV}
$$

The strength of these bounds is principally determined by the tightness of the experimental bounds on the $b_{J}^{n}$, since the difference in binding energies is close to optimal. ${ }^{3} \mathrm{He}$ is a rather weakly bound nucleus, while ${ }^{129} \mathrm{Xe}$ lies on the broad plateau of isotopes whose binding energies are close to the maximal value of $\sim 9 \mathrm{MeV}$ per nucleon.

Even if they have only order of magnitude accuracy, these are by far the best bounds on the neutron $M$ and $\Gamma$ coefficients separately. As already noted, it is possible to measure $d$ separately from $b$ in experiments with relativistic fermions. Often, this is done by measuring the relationships between energy, momentum, and velocity for extremely energetic particles. The scale of the resulting bounds is $\gamma^{-2}$, where $\gamma$ is the Lorentz factor of the particles involved. The most relativistic observable particles are cosmic ray protons near the Greisen-Zatsepin-Kuzmin limit, which have Lorentz factors of $\sim 10^{11}$. Consequently, it is impossible with direct measurements of the proton dispersion relation to place bounds on $d$ with better than $\sim 10^{-22}$ precision. Neutron measurements are far less precise, since neutrons, being unstable, are less plentiful at the highest energies. The present bounds represent an improvement over the cosmic ray limits on $d^{n}$ given in [44] of fourteen orders of magnitude.

Complementary experimental results may also be used to disentangle coefficients in the proton sector, but the results are not as good. An analysis of the $\mathrm{K} /{ }^{3} \mathrm{He}$ experiment that includes the proton polarization in the ${ }^{3} \mathrm{He}$ nucleus gives bounds on $b_{J}^{p}$ at the $10^{-31} \mathrm{GeV}$ level. However, these bounds are not independent of the neutron bounds from the same experiment. The only other bounds on this form of proton Lorentz violation are at the $10^{-27} \mathrm{GeV}$ level [33, 34]. However, some of these bounds come from a $\mathrm{H}$ maser experiment, and data collected with $\mathrm{H}$ is ideal for the kind of comparisons we have considered here, since the $\mathrm{H}$ nucleus, being a free proton, has the least possible binding energy of any isotope. Experiments with $\mathrm{H}$ also tend to be very clean, with only proton and electron coefficients involved. Combining the ${ }^{3} \mathrm{He}$ and $\mathrm{H}$ data gives disentangled bounds on the $M$ and $\Gamma$ contributions to $\tilde{b}_{J}^{p}$ at the $10^{-24} \mathrm{GeV}$ level, still significantly better than any astrophysical bound.

Improved $\mathrm{H}$ maser experiments, in connection with proton measurements made using heavier nuclei, could obviously give improved bounds on the proton $M$ and $\Gamma$ coefficients. For both the proton and neutron, there are also many combinations of Lorentz violation coefficients beyond $\tilde{b}$ which could be separated into their $M$ and $\Gamma$ parts using complementary experiments. 
In summary, we have derived new bounds on several SME coefficients in the neutron sector. All the coefficients involved have previously been bounded by atomic clock experiments, but not individually - only in particular combinations. However, when multiple measurements are available, differences in the nuclear structure of the isotopes involved make it possible to disentangle the coefficients, a fact which had not previously been appreciated. Because a weak relativistic effect in the nucleus is involved, there is a loss of precision in the disentangled bounds of at least two orders of magnitude. Nevertheless, the new bounds are at the $10^{-28} \mathrm{GeV}$ level, much better that would be possible with any other present technique.

\section{References}

[1] R. Bluhm, V. A. Kostelecký, N. Russell, Phys. Rev. Lett. 79, 1432 (1997).

[2] G. Gabrielse, A. Khabbaz, D. S. Hall, C. Heimann, H. Kalinowsky, W. Jhe, Phys. Rev. Lett. 82, 3198 (1999).

[3] H. Dehmelt, R. Mittleman, R. S. Van Dyck, Jr., P. Schwinberg, Phys. Rev. Lett. 83, 4694 (1999).

[4] R. Bluhm, V. A. Kostelecký, N. Russell , Phys. Rev. Lett. 82, 2254 (1999).

[5] D. F. Phillips, M. A. Humphrey, E. M. Mattison, R. E. Stoner, R. F. C. Vessot, R. L. Walsworth, Phys. Rev. D 63, 111101 (R) (2001).

[6] R. Bluhm, V. A. Kostelecký, C. D. Lane, Phys. Rev. Lett. 84, 1098 (2000).

[7] V. W. Hughes, et al., Phys. Rev. Lett. 87, 111804 (2001).

[8] G. W. Bennett, et al., Phys. Rev. Lett 100, 091602 (2008).

[9] R. Bluhm, V. A. Kostelecký, Phys. Rev. Lett. 84, 1381 (2000).

[10] B. R. Heckel, C. E. Cramer, T. S. Cook, S. Schlamminger, E. G. Adelberger, U. Schmidt, Phys. Rev. Lett. 97, 021603 (2006).

[11] P. Antonini, M. Okhapkin, E. Göklü, S. Schiller, Phys. Rev. A 71, 050101 (R) (2005).

[12] P. L. Stanwix, M. E. Tobar, P. Wolf, M. Susli, C. R. Locke, E. N. Ivanov, J. Winterflood, F. van Kann, Phys. Rev. Lett. 95, 040404 (2005).

[13] S. Herrmann, A. Senger, E. Kovalchuk, H. Müller, A. Peters, Phys. Rev. Lett. 95, 150401 (2005).

[14] V. A. Kostelecký, Phys. Rev. Lett. 80, 1818 (1998). 
[15] V. A. Kostelecký, Phys. Rev. D 61, 016002 (1999).

[16] Y. B. Hsiung, Nucl. Phys. Proc. Suppl. 86, 312 (2000).

[17] K. Abe et al., Phys. Rev. Lett. 86, 3228 (2001).

[18] J. M. Link et al., Phys. Lett. B 556, 7 (2003).

[19] B. Aubert et al., Phys. Rev. Lett. 96, 251802 (2006).

[20] S. M. Carroll, G. B. Field, Phys. Rev. Lett. 79, 2394 (1997).

[21] V. A. Kostelecký, M. Mewes, Phys. Rev. Lett. 87, 251304 (2001).

[22] V. A. Kostelecký, M. Mewes, Phys. Rev. Lett. 97, 140401 (2006).

[23] V. A. Kostelecký, M. Mewes, Phys. Rev. Lett. 99, 011601 (2007).

[24] F. W. Stecker, S. L. Glashow, Astropart. Phys. 16, 97 (2001).

[25] T. Jacobson, S. Liberati, D. Mattingly, Nature 424, 1019 (2003).

[26] B. Altschul, Phys. Rev. Lett. 96, 201101 (2006).

[27] B. Altschul, Phys. Rev. D 75, 041301 (R) (2007).

[28] D. Colladay, V. A. Kostelecký, Phys. Rev. D 55, 6760 (1997).

[29] D. Colladay, V. A. Kostelecký, Phys. Rev. D 58, 116002 (1998).

[30] V. A. Kostelecký, Phys. Rev. D, 69105009 (2004).

[31] V. A. Kostelecký, N. Russell, arXiv:0801.0287.

[32] C. J. Berglund, L. R. Hunter, D. Krause, Jr., E. O. Prigge, M. S. Ronfeldt, S. K. Lamoreaux, Phys. Rev. Lett. 75, 1879 (1995).

[33] V. A. Kostelecký, C. D. Lane, Phys. Rev. D 60, 116010 (1999).

[34] M. A. Humphrey, D. F. Phillips, E. M. Mattison, R. F. C. Vessot, R. E. Stoner, R. L. Walsworth, Phys. Rev. A 68, 063807 (2003).

[35] F. Canè, D. Bear, D. F. Phillips, M. S. Rosen, C. L. Smallwood, R. E. Stoner, R.L. Walsworth, V. A. Kostelecký, Phys. Rev. Lett. 93, 230801 (2004).

[36] P. Wolf, F. Chapelet, S. Bize, A. Clairon, Phys. Rev. Lett. 96, 060801 (2006). 
[37] T. W. Kornack, G. Vasilakis, M. V. Romalis, in Proceedings of the Fourth Meeting on CPT and Lorentz Symmetry, edited by V. A. Kostelecký (World Scientific, Singapore, 2008).

[38] T. Schmidt, Z. Physik 106, 358 (1937).

[39] R. Bluhm, V. A. Kostelecký, C. D. Lane, N. Russell, Phys. Rev. Lett. 88, 090801 (2002).

[40] H. Müller, P. L. Stanwix, M. E. Tobar, E. Ivanov, P. Wolf, S. Herrmann, A. Senger, E. Kovalchuk, A. Peters, Phys. Rev. Lett. 99, 050401 (2007).

[41] R. Bluhm, V. A. Kostelecký, C. D. Lane, N. Russell, Phys. Rev. D 68, 125008 (2003).

[42] V. A. Kostelecký and C. D. Lane, J. Math. Phys. 40, 6245 (1999).

[43] L. L. Foldy, S. A. Wouthuysen, Phys. Rev. 78, 29 (1950).

[44] B. Altschul, Phys. Rev. D 78, 085018 (2008). 